\title{
The Factors Affecting the Physical Activity of the Health Care Workers of Iran University of Medical Sciences: A Qualitative Study
}

\section{Soodabeh Hoveidamanesh}

Burn Research Center, Iran University of Medical Sciences

\section{Batool Tayefi}

Preventive Medicine and Public Health Research Center, Iran University of Medical Sciences

\section{Zahra Rampisheh}

Preventive Medicine and Public Health Research Center, Iran University of Medical Sciences

Narjes Khalili ( $\square$ narjes.1357@yahoo.com )

Public Responsibility in Medicine and Research https://orcid.org/0000-0001-8075-7529

\section{Mozhdeh Ramezani}

Preventive Medicine and Public Health Research Center, Iran University of Medical Sciences

\section{Research}

Keywords: physical activity, health care workers, Iran

Posted Date: July 28th, 2021

DOI: https://doi.org/10.21203/rs.3.rs-531753/v2

License: (9) This work is licensed under a Creative Commons Attribution 4.0 International License. Read Full License 


\section{Abstract}

Background: The low physical activity is a challenge in adults. A majority of adults spend many hours each week at work, so the workplace is a suitable site for health promotion and implementation of physical activity programs. This qualitative study was implemented to identify worksite physical activity barriers and facilitators from the perspective of the employees of the Iran University of Medical Science.

Methods: In the present qualitative content analysis, five focus group discussions were held with the participation of 68 personnel of Iran University of Medical Sciences. Purposive sampling with maximum diversity was carried out for the study.

Results: The analysis of the data led to the identification of three general themes, including challenges and barriers, strategies, and incentives (facilitators). The four main categories of challenges and barriers included policy-making and legislation, organizational factors, structural factors, and personal factors. Most barriers identified by the participants were placed in the personal factors and organizational factors categories. The strategies for increasing physical activity were identified in the following three categories: Policy-making and legislation, organizational factors, and environmental factors. The majority of the strategies proposed were placed in the organizational factors and policy-making and legislation categories.

Conclusions: Increasing physical activity in the workplace as a strategy for the general promotion of physical activity in people requires interventions in different areas, especially with regard to organizational factors and policy-making and legislation.

\section{Introduction}

Any body's movement, with energy expenditure, is defined as Physical activity (PA) (1). Physical activity improves muscular and cardiorespiratory fitness and bone health, As well as reduces the risk of hypertension, coronary heart disease, stroke, type 2 diabetes, various types of cancer, and depression (15). A meta-analysis indicated that workplace PA interventions improve employee's health and job stress (6). On the other hand, Physical inactivity increases no communicable diseases (NCD) by $20-30 \%$, as one of the main risk factors, and shortens individual lifespan by 3-5 years and increases the hidden cost of medical care but decreases productivity (1).

In $2016,23 \%$ of men and $32 \%$ of women above 18 years had insufficient physical activity, globally. The highest prevalence was 39\% in the WHO Region of the Americas and 35\% in the Eastern Mediterranean Region (7). In Iran, a systematic review demonstrated physical inactivity range from approximately $30 \%$ to almost 70\% (8). From 1990 to 2017, attributable disability-adjusted life years (DALY), because of low physical activity, increased 1.5 and 2-fold, globally and in Iran respectively, by the Global Burden of Disease Study (GBD) (9). 
Nevertheless, the mentioned importance, the amount of physical activity is still far from desirable, and low physical activity is still a challenge for public health (2). As physical activity may be an integral part of workplace health promotion, the workplace can be suggested, as a specially prioritized arena for this (5). according to a Working Cohort study, sickness absenteeism decreased with increased leisure time PA but increased with increased occupational PA (5).

In 2016, Iran's STEPS survey demonstrated the work (53.7\%) was the largest domain of physical activity, in all age groups and for both genders, followed by transport (33.6\%) and recreation (12.8\%), especially in 35-44 years old individuals, and men (9). On the other hand, a majority of adults spend many hours each week at work (10-12). Then the workplace was recognized as a suitable site for health promotion and raising awareness of the risk factors for NCD and implementation of Physical activity (PA) programs (6, 10-12). Several studies have also suggested that workplace interventions, for example, promoting stair use, reduce sedentarily, and increase PA behaviors at work, and all aspects of daily life $(11,12)$.

It is clear, that implementing workplace health programs can significantly improve the health status of participating employees, however, that the development and implementation of workplace health programs requires a strong commitment of organizational leadership, an inclusive health culture, and the availability of the necessary resources and infrastructure (13). Thus, the importance of organizational support strategies has been emphasized $(12,14,15)$. Because organizations are significantly different, there is a need for flexibility in creating an effective health plan according to the needs of employees (13).

In 2019-2020, a study was designed, developing the strategies for increasing the physical activity of the employees of Iran University of Medical Sciences. This qualitative study was implemented as a part of that study, to identify worksite PA barriers and facilitators from the perspective of the employees.

\section{Methods}

\section{Research methodology and paradigm}

The present qualitative study is based on naturalistic philosophy and an interpretive paradigm and uses content analysis to analyze the data. This method seeks to understand and discover the world of human experiences, since the structure of truth for each person is shaped by his own experiences (16).

The content analysis method is used in the subjective interpretation of the content of textual data. The five-stage analysis method proposed by Graneheim and Lundman was used for analyzing the content of the qualitative data. These stages included the transcription of each interview immediately afterwards, reviewing the entire text to obtain a general understanding of its content, determining the meaning units and initial codes, the classification of similar codes in more comprehensive categories, and determining the main theme of the categories (17).

\section{Research team members}


The researchers were specialists in preventive medicine and community medicine, and two of the team members held the focus group discussions and coordinated the sessions.

\section{Participants' details}

The present study was conducted with the participation of 68 people from different departments of Iran University of Medical Sciences, including physicians, nurses, health service providers, headquarter staff, managers, and specialists. The participants were selected from those who were good at expressing their ideas, had intrinsic interest in expressing their experiences, and had fairly recent and recallable experience about the situation under study (18). A face-to-face interview approach was adopted for the focus group discussions. No one withdrew from the study. The sampling process continued until the saturation of data, when no further codes could be extracted.

\section{Study setting}

The focus group discussions were held in Malard Healthcare Network, the West Healthcare Center, Hazrate Rasool Hospital, Hasheminejad Hospital and the headquarters of Iran University of Medical Sciences. The interviews were held during work hours in participants' workplace between 31.8.2019 and 29.9.2019.

\section{Data collection}

After obtaining permission from the authorities, letters were sent to the directors of the centers and hospitals, asking them to send out the invitations, which explained the study objectives, questions to be posed in the meetings, and an application for participation in the study, to any eligible candidates. Arrangements were then made and the interviewers went to the specified locations. Data were collected using focus group discussions and semi-structured open-ended questions. Data were extracted from the participants with maximum diversity in terms of demographic details and job categories, irrespective of their previous level of physical activity. The interview questions were based on a guide prepared for this purpose through a review of literature. Pilot interviews were conducted with three of the experts and the defects of the interview guide were resolved by the study team. Probing questions were also asked in the interviews if required, such as "Could you explain further?", or "What do you mean by saying ...?"

The interviews were held in participants' workplace in quiet and comfortable rooms. After introducing herself and her colleagues, explaining the study objectives and ensuring of the confidentiality of the data, and obtaining permission from the participants, the facilitator conducted the interviews. In each session, one person acted as the administrator and interviewer, and another person took notes. Before asking the questions, the participants were asked to read the informed consent form and sign it if they wished to participate in the study. At the end, the participants were asked to discuss anything else they had to say. With the participants' permission, the interviews were recorded. Each group discussion session lasted a minimum of 67 minutes and a maximum of 138 minutes.

\section{Sampling and sample size}


Non-randomized purposive sampling was performed and the study continued until the saturation of data, when no further new codes could be extracted from the group discussions. A total of 51 participants (76.1\%) were female. To observe the maximum diversity, the participants were selected from different job categories.

\section{Data analysis}

Data were analyzed through the content analysis method concurrently with their collection. This method has a major role in information management, identifying and resolving problems and shortcomings, and making decisions (19). The group discussions' contents were transcribed by a professional transcription company. The transcribed data were reviewed line by line for the analysis. Then, based on a proper schedule, the two researchers (S. H. and N. Kh.) carefully reviewed each interview text line by line at least twice and checked it against the recorded content to find the meaning units in the participants' narratives. The meaning units were encoded and were then classified, combined, and summarized based on their conceptual similarities. The extracted codes were discussed with the other researchers over two sessions. This process continued until the codes, subthemes, and main themes of the research were formed.

\section{Data rigor}

Two of the researchers and an expert in qualitative research confirmed the reliability of the data by controlling the study process. The interview texts and the initial codes extracted were sent to the participants on which to comment and thus reinforce the transferability of the extracted data.

To ensure the trustworthiness and credibility of the data, a summary of the issues discussed about each question was offered for the participants to approve. To ensure confirmability, efforts were made to not allow the researchers' assumptions to interfere with the process of data analysis. To ensure the transferability of the results and assess the validity of the data, effective communication based on trust (by explaining the study objectives clearly and ensuring the confidentiality and anonymity of the data) was established with the participants. Moreover, the participants were selected from various job categories.

\section{Results}

Five focus group discussion sessions were held with the participation of the personnel of Malard Healthcare Network, the West Healthcare Center, Hazrat-e Rasool Hospital, Hasheminejad Hospital and the staff of Iran University of Medical Sciences. A total of 68 people with a mean age of $40.7 \pm 7.29$ years participated in these sessions. Mean duration of the meetings was $95 \pm 35.76$ minutes. Table 1 presents participants' demographic details. The analysis of the data led to the identification of three general themes, including challenges and barriers, strategies, and incentives (facilitators), which are separately presented and explained in Table 2-4.

\section{- Challenges and barriers}


This theme included four subthemes and 37 codes. The main four subthemes of challenges and barriers were policy-making and legislation, organizational factors, structural factors, and personal factors.

To identify the largest possible number of barriers to physical activity from the participants' views, the number of times each code in the barrier theme was repeated was counted. The majority of the codes pertained to the subtheme of personal factors, followed by organizational factors and structural factors. Only a few of the codes pertained to the subtheme of policy-making and legislation. Figure 1 presents the repetition of the codes in this theme.

\section{- Policy-making and legislation}

In the policy-making and legislation subtheme, some of the participants believed that the personnel's physical activity not being a priority for the managers and their being non-compulsory were an important barrier. "The main problem is that there's no compulsory law on it. There must be compulsion in anything you want to motivate people to do; like, we have to work because it's the law and we have to work to get paid" (P9).

The participants believed that the authorities' priority was working, and the personnel's physical activity and physical and mental health play no part in the job policies.

"The priority of the unit managers is working, and not physical activity. If there is some compulsion, I mean, if a value system is defined or but if a payment would be allocated to exercise on the payment scale" (P2).

\section{- Organizational factors}

Regarding organizational factors, the most frequently-discussed items were organizational culture, type of desk work, heavy workloads, and large number of shifts. Moreover, long work hours, the shortage of workforce, and being assigned multiple roles in the system were other issues emphasized by the participants.

"I personally believe that the thing that has led me to have less physical activity is that my work in this job and my work hours are excessive, and since I get very tired, when I finally get to leave work, I can't handle much physical activity anymore" (P17).

\section{- Structural factors}

The main barriers in the subtheme of structural factors that were emphasized by the participants included the lack of physical space for exercise or the poor conditions of the physical space for physical activity both size-wise and quality-wise and also the distance from home to the gym. The challenges of performing physical activity for women, especially married women, comprised some other reasons for inactivity in this category. 
"If we want to use the gym facilities at the university after work, since the space is small, we have to wait for our turn on the machines" (P60).

"For example, if they said that we should gather round in the praying room every Monday to both have a chat and exercise in between, we would have gone, but we were face with limitation. Well our praying room is not just a praying room; it is also the lecture theater, the conference room, and the praying room. For instance, whenever we went there to exercise, others would want to come in after us and do their praying" (P2).

"Well, most staff in health setting are women, married, and have kids, like myself. With kids, when I go home after work I have to pick the kids up from kindergarten or school, and then do the house work. I have to handle all these, and then if I want to spend some time exercising, which I like very much, can I? I love going to the gym, I might even plan for it, but I can't really manage it" (P6).

\section{- Personal factors}

In the subtheme of personal factors, the main barriers extracted in the present study included the shortage of time, physical and mental exhaustion, the lack of motivation, and financial problems.

"I think the main thing that makes us have no physical activity at all is the multiplicity of tasks assigned to us that fall beyond our field of work. We have forgotten ourselves altogether; we don't show love toward ourselves at all, as if we do not pay attention to ourselves. Our seniors don't care about us at all, like, do they ever ask if their personnel be physically and mentally healthy or not? We feel we have been forgotten" (P19).

\section{- Strategies}

The strategies for promoting physical activity were classified into three subthemes and 19 codes, including policy-making and legislation, organizational factors, and environmental factors.

\section{- Policy-making and legislation}

The findings related to this subtheme emphasized the development and implementation of exercise breaks, allocating funds to the personnel's physical activity at the macro level, and raising awareness and engaging the managers and officials.

"There must be a law for this in the country, like the breastfeeding break that makes the employees actually get up and go to breastfeed their child. A period of, like, half an hour or twenty minutes should be legislated for this, and it should become the law, forcing employees to take it" (P14).

"There should be a budget specifically allocated for exercise, so we can create a space for our colleagues. We must first have the space" (P15). 
"When the organization and senior managers understand the necessity of it and if I don't have to face the ramifications of not being at my desk for half an hour from ten to ten thirty [that would be good]; I mean, if I don't have to be stressed over not being at my desk if my direct manager contacts me when I'm not at my desk" (P52).

\section{- Organizational factors}

The strategies emphasized by the participants included needs assessment and assigning a sports liaison, recruiting a sports coach to teach the right exercises according to the people's physical conditions, increasing the workforce to reduce work pressure and stress, creating opportunities for performing physical activities and reforming the organizational culture.

\section{Needs assessment and assigning a sports liaison}

"For general and specialized exercises, I think there should first be a responsible and expert liaison to assess the exercise needs of the personnel and see what their general or specialized needs are, and then based on those needs, the expert liaison should interview the people one by one; perhaps one person is incapable of performing many of the exercises and they are harmful for her" (P10).

\section{Recruiting a coach}

"A particular exercise is suitable for each person depending on his/her physical conditions, and the coach should advise the individuals on which physical activities to perform" (P59).

\section{Increasing the workforce}

"As we said, we are short of workforce. We should reduce our work hours, so we have time for exercise. When I do not have the ability and strength, I cannot come first to exercise" (P11).

\section{Reforming the organizational culture}

"I think the organizational culture should be reformed, and then we must definitely have a personnel health record. I mean, each person should consider it their obligation, so that he can monitor his progress, in terms of evaluation, and think about exercise physically and mentally, whether during work hours or outside the work hours, and now, the workload should be somewhat adjusted too" (P9).

\section{- Environmental factors}

The results in this section emphasized the need for diversity in physical activities, forming sports teams, creating a suitable, standard and safe place for all the personnel, especially women, and easy access to these places.

\section{Diversity in physical activity and forming sports teams}


"Team sports and group activities are good and make the place energetic" (P9).

"Team sports are motivating. To guide these, you should form groups and networks, make sports networks, and then choose one person as the leader to guide the rest along" (P68).

\section{Creating an appropriate place with easy access}

"I think the university should dedicate a place, be it a gym, a pool, some place, where everyone can use for free or for a small amount of money, and if they have these facilities, everyone can use them and do their exercise there. Signing contracts with gyms close to each hospital or close to where the personnel live is also good" (P46)

\section{- Motivators (Facilitators)}

The theme of motivators led to the extraction of organizational and motivational subthemes, with seven and 13 codes, respectively.

\section{- Organizational factors}

Most participants believed the main facilitators of physical activity to include the organization paying fitness subsidies, including the personnel's physical activity in their performance evaluation, and building a healthy fitness culture.

\section{Paying fitness subsidies and drawing contracts with gyms and pools}

"Paying fitness subsidies by drafting contracts with gyms and pools and giving the staff passes or subsidies, and expanding the university gymnasium" (P60).

"If they draft a contract with some place and tell us that both men and women can go on certain days without restrictions on time, I think it will be welcomed by all the staff. But there is always the issue of the costs, and we don't expect it to be free of charge, just at a lower cost" (P46).

"The staff should be given passes to various gyms for free or half price, which should be financed by the ministry and not the network. There should be different places across Tehran, they should be obligatory, and the staff should be forced by the directors to use them, and subsidies should be paid to motivate the personnel" (P2).

\section{Including the personnel's physical activity in their performance evaluation}

"An evaluation system should be defined for this, because there's currently nothing in the civil service law at all; a payment should be considered for exercising on the payment scale; institutionalizing exercise, laying the groundwork for it and building a culture of physical activity are key. Our civil service law should be revised" (P11). 
"It has to do with the culture. Generally, we don't feel the need neither at school nor in the family to perform physical activities. You grow up in this way; you go to college and then to the workplace in this way, and then if one day they give me this opportunity [to exercise regularly], I don't welcome it much. It has partly to do with the culture; its necessity has not been internalized. Now building a culture of physical activity is associated with knowledge. If I don't know how to sit correctly, then I don't sit correctly. It is the same with walking; I don't walk correctly if I don't know how to" (P55).

\section{- Motivational factors}

The participants believed that building and improving motivation are key components of encouraging physical activity. Material and immaterial incentives, holding competitions in fields such as mountainclimbing, hiking and nature tours with the family were some of the discussed measures.

\section{Material and immaterial incentives}

"Giving points for exercise. Giving points to someone who exercises rather than deducting points from someone who doesn't exercise" (P60).

\section{Holding competitions}

"Another issue is that competitions should be ongoing. We only have few different occasions on which competitions are held, such as the anniversary of the Islamic Revolution, the ten-day celebration of Khomeini's return to Iran, the government week, etc.; however, if these were ongoing, like, if they were seasonal, in all disciplines, then I think it would be motivating. The personnel should all be somehow allowed to take part in these competitions or hiking tours; they should be performed in the same way with fairness, so that everyone can take part" (P4).

\section{Mountain-climbing, hiking, and nature tours with the family}

"Creating a family atmosphere so that family members and friends can also attend these sports activities and turning them into group activities will certainly make them more effective" (P50).

\section{Discussion}

The present qualitative study was conducted to identify the factors affecting the physical activity of the personnel of Iran University of Medical Sciences, so that they can be used in designing an intervention to promote physical activity among the university personnel. The content analysis of the interviews led to the identification of three main themes, including challenges and barriers, strategies, and incentives (facilitators).

Challenges and barriers were categorized as policy-making and legislation, organizational factors, structural factors, and personal factors. In previous studies, barriers have been categorized into groups such as organizational views, operational outlook, and personal views (12) or into physical, psychological 
and environmental dimensions (6). The various qualitative studies on this subject have found similar themes for the barriers to physical activity in the workplace, aside from these categories. The participants of the present study considered the shortage of time, physical exhaustion, and the lack of a suitable space for exercise as the biggest barrier to their physical activity, with the first two items being placed in the theme of personal factors. In other studies, the greatest barriers to exercise were noted as "having to invest time", "fatigue" (20), "physical limitations due to pain and frailty", "lack of motivation", "lack of time", and "job commitment" (6), "excessive exhaustion", and "work commitment/long work hours" (21). In some studies, the greatest barriers differed depending on the job category or type of work. For example, in one study, the managers recalled structural/organizational barriers, including regulations, costs, and the competitive aspect of work, while the employees tended to focus on personal limitations, such as the time and physical place for exercise (10). As another example, employees in the transport industry, who are at a greater risk of inactivity compared to other jobs, regarded the changes in their work schedule, bad weather conditions, and the lack of planned holidays as the main barriers to their physical activity (22). The barriers and facilitators of physical activity for the midwives working in Scotland hospitals and health centers included fatigue, stress, family responsibilities, unpredictable rest hours and the work shifts (23). These items were more or less discussed in the same way by the hospital staff in the present study. Some considered organizational culture as a barrier to physical activity in the workplace, which emphasizes the need for considering particular cultural barriers and other barriers in the workplace just as in the study by Cooper et al. (24). Similar to previous studies (6), psychosocial and environmental barriers appear to be a greater obstacle to physical activity than physical disorders.

Incentives (facilitators) were placed into organizational and motivational categories. In previous studies, the most powerful incentives for physical activity consisted of family relationships, social support, and the perceived health benefits of physical activity (6), social support, subsidies for classes, and breaks given at specific times (23). The environmental background and resources of the workplace can be both a barrier and a facilitator of physical activity (23). In the present study, creating a space for exercise with family and friends was cited as a factor for increasing the motivation to exercise. Moreover, organizational incentives such as the payment of subsidies by the organization for exercise classes, including the personnel's physical activity in the performance evaluation system, laying the groundwork and building a culture of exercise as well as creating and promoting motivation were regarded as the key incentives for performing physical activity. The results obtained by Brakenridge et al. show that workplace interventions supported by the organization are acceptable and can lead to long-term changes in awareness and culture (14). Moreover, evidence-based interventions, supported by key individuals as role models, can spread to other workplaces (14). Similar to in previous studies (25), some of the participants in the present study stated that the design of urban areas should facilitate access to suitable spaces for playing sports in cities.

In the present study, the strategies for increasing physical activity were placed in three Subthemes, including policy-making and legislation, organizational factors, and environmental factors. In Planchard's study, interventions were categorized as preparation, empowerment, reinforcement, environmental factors and policies (6).

Page $11 / 21$ 
Worksite wellness programs as the promotion of physical activity can help businesses creating effective policies and programs to meet employee and business priorities (11). In agreement with previous studies (23), the present study showed that interventions should focus on improving interpersonal relationships, reducing workplace stresses, and increasing social support. Just like previous studies (26), the present findings emphasized the need to pay further attention to women, especially working mothers, as the needs of this group should be further addressed by health counseling (26).

Although most barriers discussed by the participants were placed in the personal factors category, the majority of the strategies were related to organizational factors and policy-making and legislation categories. For instance, the lack of awareness about the risks of inactivity, which is in the personal factors category of the theme of barriers, can be resolved by holding physical activity training courses, which is in the organizational factors category of the theme of strategies. Thus, as noted, various studies, including the present one, have emphasized the importance of organizational support strategies $(12,14$, $15)$, even though various studies have discussed organizations' shortfalls in addressing these issues. For instance, in a study by Bailey et al. on the policies supporting physical activity in the workplace, a small number of the examined organizations had a written policy for increasing physical activity and allocating time to exercise during work hours, and the lack of such policy was described by the respondents as a barrier to greater physical activity in the personnel (11). In another study, Chau et al. investigated the views of the personnel of 12 different organizations and concluded that physical activity has not been a priority in the occupational health programs of organizations, although prioritizing physical activity in these programs is necessary for creating physical activity-friendly workplaces (10). It must be supported at all levels in an organization to make physical activity an integral part of daily work (10).

The limitations of this study included not holding individual interviews alongside the group discussions, which could have provided more information about the personal barriers to physical activity, and this study assessed the views of the personnel only through group discussions, which could be a limitation. Meanwhile, holding several sessions in different university departments aimed at covering a maximum diversity of views and examining the views of administrative, health, medical and headquarter staffs were among the strengths, which somewhat made up for the discussed limitation.

\section{Conclusion}

Reviewing the results of other qualitative studies shows that similar to the present study barriers and facilitators are in different domains, so interventions are required in different areas as a strategy to increase physical activity in the workplace, especially in areas related to organizational factors and policy-making and legislation. The researchers therefore recommend developing a written program to address the lack of physical activity in university staff with an emphasis on strategies in different areas, especially in relation to organizational factors, while also taking into account the facilitators.

An important point is that many strategies to remove the barriers can be implemented at a negligible cost, as they require only the attention of the staff and officials. 


\section{Abbreviations}

PA: Physical Activity

\section{Declarations}

\section{Ethical approval and consent to practice:}

The study was conducted after obtaining permission from the university authorities and receiving a code of ethics (No: IR.IUMS.REC.1397.733) from the Ethics Committee of Iran University of Medical Sciences. While explaining the study objectives, the participants were ensured of the confidentiality of the data. Participation in the study was voluntary and contingent on receiving the candidates' written consent, and the participants were allowed to withdraw from it at any stage. The interviews were recorded with the prior permission of the participants, and all audio files were deleted after the data analysis was over.

\section{Consent for publication:}

Not applicable

\section{Availability of data and materials:}

The datasets generated and/or analyzed during the current study are not publicly available due to confidentiality but are available from the corresponding author on reasonable request.

\section{Competing interests:}

The authors declare that they have no competing interests.

\section{Funding:}

Iran University of Medical Sciences. Tehran, Iran.

\section{Authors' contributions:}

B.T and M.Z held the focus group discussions and coordinated the sessions. S. H and N. Kh carefully reviewed each interview text line by line at least twice and encoded. The extracted codes were discussed with the other researchers (Z.R, B.T, and M.Z). S. H and N. Kh were the major contributor in writing the manuscript. All authors read and approved the final manuscript.

\section{Acknowledgements:}

Not applicable

\section{References}


1. WHO. Physical activity [2020 July]. Available from: https://www.who.int/health-topics/physicalactivity.

2. Alves AJ, Viana JL, Cavalcante SL, Oliveira NL, Duarte JA, Mota J, et al. Physical activity in primary and secondary prevention of cardiovascular disease: Overview updated. World Journal of Cardiology. 2016;8(10):575.

3. Booth FW, Roberts CK, Thyfault JP, Ruegsegger GN, Toedebusch RG. Role of inactivity in chronic diseases: evolutionary insight and pathophysiological mechanisms. Physiological Reviews. 2017;97(4):1351-402.

4. McTiernan A, Friedenreich CM, Katzmarzyk PT, Powell KE, Macko R, Buchner D, et al. Physical Activity in Cancer Prevention and Survival: A Systematic Review. Medicine and science in sports and exercise. 2019;51(6):1252-61.

5. Sjøgaard G, Christensen JR, Justesen JB, Murray M, Dalager T, Fredslund GH, et al. Exercise is more than medicine: The working age population's well-being and productivity. Journal of Sport and Health Science. 2016;5(2):159-65.

6. Planchard J-H, Corrion K, Lehmann L, d'Arripe-Longueville F. Worksite physical activity barriers and facilitators: A qualitative study based on the transtheoretical model of change. Frontiers in public health. 2018;6:326.

7. WHO. Prevalence of insufficient physical activity [cited 202027 July]. Available from: https://www.who.int/gho/ncd/risk_factors/physical_activity_text/en/.

8. Fakhrzadeh H, Djalalinia S, Mirarefin M, Arefirad T, Asayesh H, Safiri S, et al. Prevalence of physical inactivity in Iran: a systematic review. Journal of cardiovascular and thoracic research. 2016;8(3):92.

9. Mohebi F, Mohajer B, Yoosefı M, Sheidaei A, Zokaei H, Damerchilu B, et al. Physical activity profile of the Iranian population: STEPS survey, 2016. BMC public health. 2019;19(1):1266.

10. Chau JY, Engelen L, Kolbe-Alexander T, Young S, Olsen H, Gilson N, et al. "In Initiative Overload": Australian Perspectives on Promoting Physical Activity in the Workplace from Diverse Industries. International journal of environmental research and public health. 2019;16(3).

11. Bailey MM, Coller RK, Porter KMP. A qualitative study of facilitators and barriers to implementing worksite policies that support physical activity. BMC public health. 2018;18(1):1-8.

12. Bredahl TVG, Særvoll CA, Kirkelund L, Sjøgaard G, Andersen LL. When intervention meets organisation, a qualitative study of motivation and barriers to physical exercise at the workplace. The Scientific World Journal. 2015;2015.

13. Cahalin LP, Kaminsky L, Lavie CJ, Briggs P, Cahalin BL, Myers J, et al. Development and implementation of worksite health and wellness programs: a focus on non-communicable disease. Progress in cardiovascular diseases. 2015;58(1):94-101.

14. Brakenridge CL, Healy GN, Hadgraft NT, Young DC, Fjeldsoe BS. Australian employee perceptions of an organizational-level intervention to reduce sitting. Health promotion international. 2018;33(6):96879. 
15. Passey D, Kavanagh L, Hammerback K, Harris J, Hannon P. Manager and supervisor support for worksite health promotion programs. Wash State Health Care Auth. 2016.

16. Speziale HS, Streubert HJ, Carpenter DR. Qualitative research in nursing: Advancing the humanistic imperative: Lippincott Williams \& Wilkins; 2011.

17. Graneheim UH, Lundman B. Qualitative content analysis in nursing research: concepts, procedures and measures to achieve trustworthiness. Nurse education today. 2004;24(2):105-12.

18. A. O. Phenomenology: A method for nursing research. Advances in nursing science. 1983;5(2):49-64.

19. Wildemuth BM. Applications of social research methods to questions in information and library science: ABC-CLIO; 2016.

20. Keohane DM, McGillivary NA, Daly B. Physical activity levels and perceived barriers to exercise participation in Irish General Practitioners and General Practice trainees. Irish medical journal. 2018;111(2):690.

21. Blackford K, Jancey J, Howat P, Ledger M, Lee AH. Peer reviewed: office-based physical activity and nutrition intervention: barriers, enablers, and preferred strategies for workplace obesity prevention, Perth, Western Australia, 2012. Preventing chronic disease. 2013;10.

22. Das BM, Petruzzello SJ. Barriers to Physical Activity in a Mass Transit Population: A Qualitative Study. Journal of physical activity \& health. 2016;13(1):53-8.

23. Holly D, Swanson V. Barriers and facilitators of midwives' physical activity behaviour in hospital and community contexts in Scotland. Journal of advanced nursing. 2019;75(10):2211-22.

24. Cooper K, Barton GC. An exploration of physical activity and wellbeing in university employees. Perspectives in public health. 2016;136(3):152-60.

25. Marconnot R, Marín-Rojas AL, Delfa-de-la-Morena JM, Pérez-Corrales J, Gueita-Rodríguez J, Fernández-de-Las-Peñas C, et al. Recognition of Barriers to Physical Activity Promotion in Immigrant Children in Spain: A Qualitative Case Study. International journal of environmental research and public health. 2019;16(3).

26. Dombrowski JJ. Barriers to physical activity among working mothers. AAOHN journal : official journal of the American Association of Occupational Health Nurses. 2011;59(4):161-7.

\section{Tables}

Table 1: Demographic characteristics of participants $(n=68)$ 


\begin{tabular}{|c|c|c|}
\hline Variable & & Number (\%) \\
\hline \multirow[t]{2}{*}{ Gender } & Male & $16(23.9 \%)$ \\
\hline & Female & $51(76.1 \%)$ \\
\hline \multirow[t]{2}{*}{ Marital status } & Married & $40(61.5 \%)$ \\
\hline & Single & $24(36.9 \%)$ \\
\hline \multirow[t]{5}{*}{ Education } & Below high school diploma & $1(1.5 \%)$ \\
\hline & High school diploma \& associate degree & $7(10.6 \%)$ \\
\hline & Bachelor's degree & $29(43.9 \%)$ \\
\hline & Master's degree & $18(27.3 \%)$ \\
\hline & $\mathrm{PhD}$ & $11(16.7 \%)$ \\
\hline \multirow[t]{3}{*}{ Work shifts } & Day & $53(84.1 \%)$ \\
\hline & Night & $1(1.6 \%)$ \\
\hline & Rotating & $9(14.3 \%)$ \\
\hline \multirow[t]{4}{*}{ Job category } & Health & $28(46.7 \%)$ \\
\hline & Medical & $16(26.7 \%)$ \\
\hline & Administrative/financial & $12(20 \%)$ \\
\hline & Faculty member & $4(6.7 \%)$ \\
\hline
\end{tabular}

Table 2: The codes extracted for the challenges and barriers theme 
Theme

Challenges and barriers
Sub theme

1.1. Policy-making and legislation

1.2. Organizational factors
1.3. Structural factors

\section{Code}

1.1.1 Employees' physical activity not being a priority at the macro level

1.1.2 Physical activity not being compulsory

1.2.1. Long work hours

1.2.2. Large number of work shifts

1.2.3. Playing multiple roles in the system

1.2.4. Shortage of workforce

1.2.5. Mechanization and computerization of tasks

1.2.6. Allocating a bad time to exercise (after work hours)

1.2.7. Type of work (working at a desk or computer)

1.2.8. Stressful work environment

1.2.9. Ruling organizational culture

1.2.10. Personnel's physical and mental health not being important for some managers

1.2.11. Job burnout

1.2.12. Poor notifications about the university's sports and recreational programs

1.2.13. The lack of motivation for physical activity

1.3.1. The lack of physical space for exercise

1.3.2. Distance (from work to home or home to the gym)

1.3.3. Poor physical space unsuitable for performing physical activity (quality and size-wise)

1.3.4. The lack of shower facilities at the university gym

1.3.5. Old equipment and machines

1.3.6. Lack of a coach

1.3.7. The allocation of morning hours to women and evenings to men

1.3.8. Marriage and raising children

1.3.9. Personal and family culture

1.3.10. Female gender

1.3.11. The small space at home and the limitations of 
performing exercise in apartments

\subsection{Personal factors 1.4.1. The lack of motivation}

1.4.2. Exercise not being a priority in life

1.4.3. Physical exhaustion

1.4.4. Mental exhaustion

1.4.5. Shortage of time (time constraints)

1.4.6. Personal culture

1.4.7. Having no exercise plans

1.4.8. Financial and livelihood problems

1.4.9. Laziness

1.4.10. Family responsibilities

1.4.11. The lack of awareness about the harms of a sedentary lifestyle

Table 3: The codes extracted for the strategies theme 
Strategies 2.1. Policy-making and legislation

2.2. Organizational factors
2.3. Environmental factors
2.1.1. Creating and enforcing exercise breaks

2.1.2. Allocation of budget to personnel's physical activity at the macro level

2.1.3. Raising awareness and engaging managers and officials in physical activity

2.1.4. Making exercise mandatory for the personnel

2.1.5. Incorporating physical activity into the civil service law

2.1.6 Training and exercise programs in the workplace

2.1.7. Promoting a culture of physical activity from childhood

2.2.1. Needs assessment and assigning an exercise liaison

2.2.2. Hiring a coach

2.2.3. Increasing the workforce

2.2.4. Reducing the work hours

2.2.5. Holding physical activity training

2.2.6. Reforming the organizational culture about the personnel's physical activity

2.2.7. Facilitating the health personnel's transportation to and from the university gym

2.2.8. Allowing different time slots for physical activity (before, during, and after work)

2.2.9. The use of city bikes at the university, and morning jogs in groups

2.2.10. Installing workplace exercise software on the personnel's computers

2.3.1. Diversity in physical activity, and creating a suitable space for exercise

2.3.2. Creating a suitable space for exercise, especially for women

2.3.3. Standard, safe, easily-accessible exercise spaces

Table 4: The codes extracted for the facilitators theme 
Incentives

(facilitators)
3.1.

Organizational factors
3.2.

Motivational factors
3.1.1. Giving gym passes or paying fitness subsidies and drafting contracts with gyms and pools

3.1.2. The inclusion of physical activity in the personnel's performance evaluation system

\subsubsection{Laying the groundwork}

\subsubsection{Internalization and building a culture of exercise}

3.1.5. Inclusion of physical activity in the personnel's health records

3.1.6. Paying for exercise classes through the personnel's wages

3.1.7. Increasing the number of university gyms and sports facilities and improving the quality of the current gyms

\subsubsection{Material and immaterial incentives}

3.2.2. Physical activities in teams and groups

3.2.3. The personnel going on walks in parks

3.2.4. Holding ongoing and seasonal competitions

3.2.5. Physical activity promoting campaigns

3.2.6. Proper announcement of upcoming sports activities

3.2.7. Creating desire and sensitivity

3.2.8. Ensuring equity among the personnel from different departments

\subsubsection{Using NGOs for support}

3.2.10. Distributing educational pamphlets in the workplace to encourage the personnel to perform physical activity

\subsubsection{Diversity of sports fields}

3.2.12. Holding mountain climbing, hiking, and nature tours with the personnel's family

3.2.13. Hanging inspirational messages and quotes based on scientific evidence on the office walls to encourage physical activity 


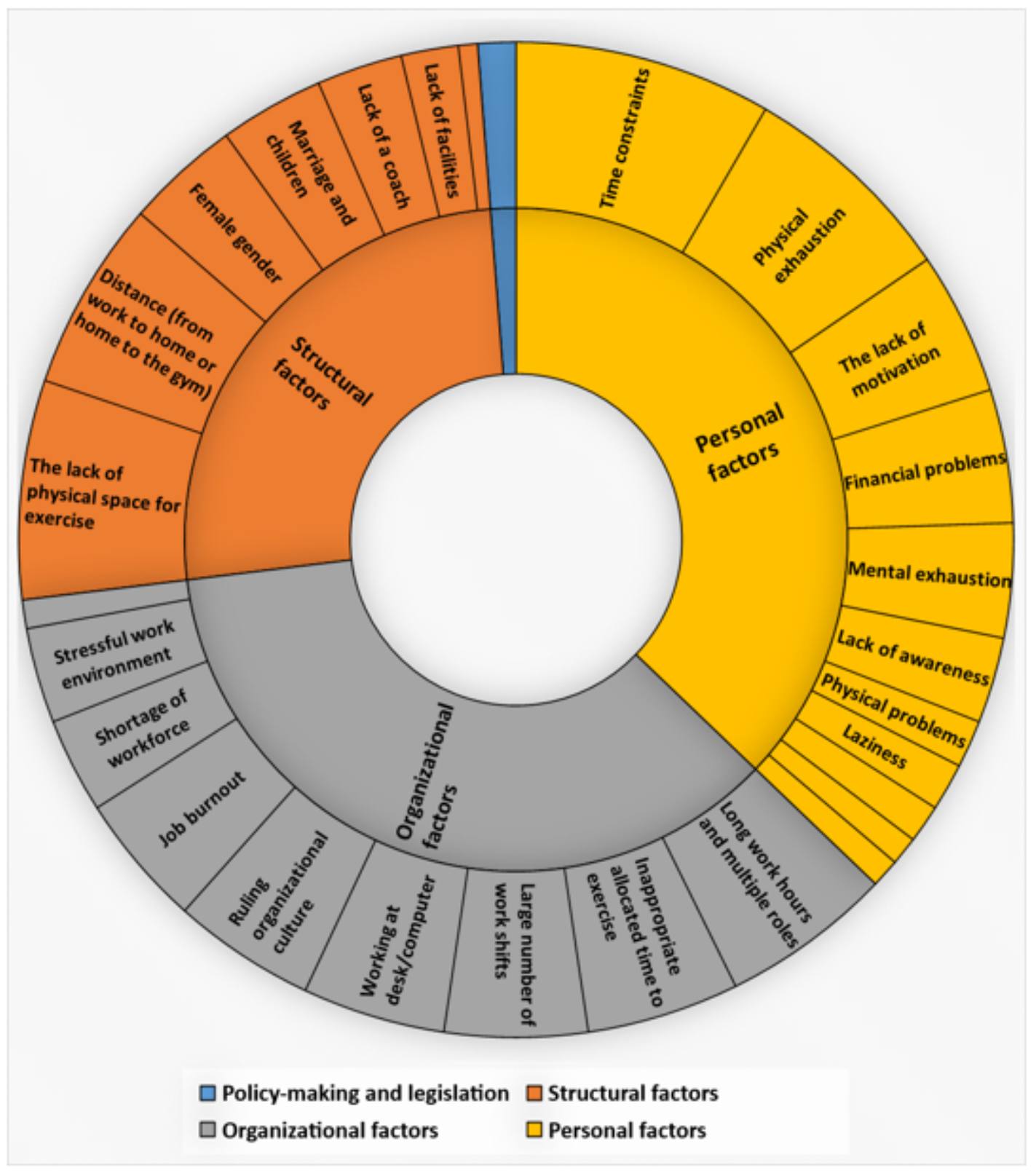

Figure 1

The most frequent extracted codes for the challenges and barriers theme 